\title{
Performance of the recommended ESC/
} EASD cardiovascular risk stratification model in comparison to SCORE and NT-proBNP as a single biomarker for risk prediction in type 2 diabetes mellitus

Suriya Prausmüller ${ }^{1}$, Michael Resl ${ }^{2}$, Henrike Arfsten ${ }^{1}$, Georg Spinka ${ }^{1}$, Raphael Wurm', Stephanie Neuhold ${ }^{3}$, Philipp E. Bartko ${ }^{1}$, Georg Goliasch', Guido Strunk ${ }^{4}$, Noemi Pavo ${ }^{1 *}$, Martin Clodi ${ }^{2}$ and Martin Hülsmann ${ }^{1}$

\begin{abstract}
Background: Recently, the European Society of Cardiology (ESC) and European Association for the Society of Diabetes (EASD) introduced a new cardiovascular disease (CVD) risk stratification model to aid further treatment decisions in individuals with diabetes. Our study aimed to investigate the prognostic performance of the ESC/EASD risk model in comparison to the Systematic COronary Risk Evaluation (SCORE) risk model and N-terminal pro-B-type natriuretic peptide (NT-proBNP) in an unselected cohort of type 2 diabetes mellitus (T2DM).

Methods and results: A total of 1690 T2DM patients with a 10-year follow up for fatal CVD and all-cause death and a 5-year follow up for CVD and all-cause hospitalizations were analyzed. According to ESC/EASD risk criteria 25 (1.5\%) patients were classified as moderate, 252 (14.9\%) high, 1125 (66.6\%) very high risk and 288 (17.0\%) were not classifiable. Both NT-proBNP and SCORE risk model were associated with 10-year CVD and all-cause death and 5-year CVD and all-cause hospitalizations while the ESC/EASD model was only associated with 10-year all-cause death and 5-year all-cause hospitalizations. NT-proBNP and SCORE showed significantly higher C-indices than the ESC/EASD risk model for CVD death [0.80 vs. $0.53, p<0.001 ; 0.64$ vs. $0.53, p=0.001]$ and all-cause death $[0.73,0.66$ vs. $0.52, p<0.001$ for both]. The performance of SCORE improved in a subgroup without CVD aged 40-64 years compared to the unselected cohort, while NT-proBNP performance was robust across all groups.
\end{abstract}

Conclusion: The new introduced ESC/EASD risk stratification model performed limited compared to SCORE and single NT-proBNP assessment for predicting 10-year CVD and all-cause fatal events in individuals with T2DM.

Keywords: Diabetes mellitus, Cardiovascular risk, Prevention

*Correspondence: noemi.pavo@meduniwien.ac.at

${ }^{1}$ Department of Internal Medicine II, Medical University of Vienna, Waehringer Guertel 18-20, 1090 Vienna, Austria

Full list of author information is available at the end of the article

\section{Background}

Diabetes is associated with a substantially increased risk to develop cardiovascular disease (CVD) [1]; however, as individuals with diabetes represent a highly heterogeneous population, incremental CVD risk is not equally distributed among diabetic patients [2]. Therefore, the development of individualized CVD risk assessment tools is essential to warrant a personalized therapy approach.

c) The Author(s) 2021. This article is licensed under a Creative Commons Attribution 4.0 International License, which permits use, sharing, adaptation, distribution and reproduction in any medium or format, as long as you give appropriate credit to the original author(s) and the source, provide a link to the Creative Commons licence, and indicate if changes were made. The images or other third party material in this article are included in the article's Creative Commons licence, unless indicated otherwise in a credit line to the material. If material is not included in the article's Creative Commons licence and your intended use is not permitted by statutory regulation or exceeds the permitted use, you will need to obtain permission directly from the copyright holder. To view a copy of this licence, visit http://creativeco mmons.org/licenses/by/4.0/. The Creative Commons Public Domain Dedication waiver (http://creativecommons.org/publicdomain/ zero/1.0/) applies to the data made available in this article, unless otherwise stated in a credit line to the data. 
Recently, the European Society of Cardiology (ESC) in collaboration with the European Association for the Study of Diabetes (EASD) published new guidelines for the prevention and management of CVD in patients with prediabetes and diabetes [3]. For the first time, the use of a CVD risk stratification model is recommended to aid treatment decisions in individuals with diabetes. The ESC/EASD risk model stratifies diabetic patients into three different risk categories based on the 10-year risk estimate for fatal CVD adapted from the 2016 European Guidelines on CVD prevention in clinical practice [4]. To the best of our knowledge, the predictive performance of the newly introduced risk stratification model has not been verified in individuals with diabetes.

The Systematic COronary Risk Evaluation (SCORE) equation is commonly used for estimating the 10-year risk of fatal CVD in the general population [5]. In the original publication of the SCORE project, it has been suggested that SCORE based risk assessment could be used for a rough assessment of CVD risk in diabetic patients. Yet, the predictive performance of SCORE has not been tested in patients with long-standing diabetes specifically [6]. As a result, its application for risk estimation in individuals with diabetes cannot be recommended [4].

The prognostic value of $\mathrm{N}$-terminal pro-B-type natriuretic peptide (NT-proBNP) for CVD outcomes in patients with diabetes has been demonstrated and was confirmed in numerous studies [7-24]. Nonetheless, the new guidelines do not recommend routine assessment of circulating biomarkers for CVD risk estimation in diabetic patients [3].

This study aimed to perform a head-to-head comparison of the predictive performance of the ESC/EASD model against SCORE and NT-proBNP for risk assessment of 10-year CVD death and all-cause death (i) in an unselected type 2 diabetes mellitus (T2DM) cohort, (ii) in selected patients with T2DM with characteristics similar to the SCORE derivation cohort and (iii) to investigate the outcome-specific performance of the different risk estimates. Additionally, the prognostic utility of the risk assessments for 5-year CVD and all-cause hospitalization was evaluated.

\section{Methods}

\section{Study population}

From December 2005 through January 2010 a total of 2186 patients with T2DM from 4 diabetes outpatient clinics were included in a prospective registry. Medical history, including comorbidities, diabetes duration, medical therapy and assessment of risk factors, was recorded at enrolment. Patients were followed up as clinically appropriate. All patients gave written informed consent. The study was approved by the local Ethics Committee of the Medical University of Vienna and complies with the principles of the Declaration of Helsinki.

\section{Laboratory analysis}

Blood samples were collected under fasting conditions and immediately sent to the local laboratory. Estimated glomerular filtration rate (eGFR) was assessed by the Modified Diet for Renal Disease Study equation. NTproBNP determination was performed directly using the cobas $\mathrm{h} 232$ point-of-care analyzer by Roche (Roche Diagnostics, Basel, Switzerland) with a lower detection limit of $59 \mathrm{pg} / \mathrm{ml}$. Within-series coefficients of variation ranged from 4.8 to $14.8 \%$ as previously determined by Bertsch et al. [25] Urine albumin/creatinine ratio was assessed quantitively in fresh spot urine samples according to the local laboratory standards.

\section{Calculating risk estimates}

\section{a. ESC/EASD risk stratification model}

Patients were categorized as moderate, high and very high risk based on the predicted 10-year risk estimates for CVD death $<5 \%, 5-10 \%$ and $>10 \%$, respectively, according to the ESC/EASD cardiovascular risk categories as indicated in Additional file 1: Table S1. [3] Except for renal impairment no precise definition on the rating of the respective risk factors used within the ESC/EASD stratification model was given. Thus, we defined age, obesity and proteinuria as being at risk $>50$ years, $\geq 30 \mathrm{~kg} / \mathrm{m}^{2}$ and with a urinary albumin/creatinine ratio $>30 \mathrm{mg} / \mathrm{mmol}$, respectively. High blood pressure and dyslipidemia were defined according to the criteria of the respective current European guidelines [26, 27], as documented in medical charts or on specific therapy. Smoking status was assessed based on hospital charts and by self-report. CVD diagnosis was defined with a corresponding main diagnosis according to at least one of the following International Statistical Classification of Diseases and Related Health Problems 10th Revision (ICD-10) codes: I21-I25 (ischemic heart diseases), I63-I66 (cerebral artery disease), I70-I74 (peripheral artery disease), I44.7 (left bundle-branch block), I50 (heart failure) I48 (atrial fibrillation and flutter), I11 (hypertensive heart disease) and I34-I36 (valve disorders). As retinopathy and left ventricular hypertrophy were not systematically assessed, these variables were not included in the analysis. Additional file 1: Table S2 provides an overview of the specific cut-offs 
and definitions used for risk stratification of the ESC/ EASD model.

b. SCORE risk model

SCORE risk estimation is recommended for individuals without CVD and aged 40-64 years in accordance to the selection criteria of SCORE $[4,5]$. The 10-year fatal CVD risk was calculated using the low SCORE risk chart based on the risk variables age, sex, smoking status, total cholesterol and systolic blood pressure [5]. As indicated in the reference publication, we multiplied the SCORE risk estimates by 2 for men and 4 for women to account for the increased $\mathrm{CV}$ (cardiovascular) risk in individuals with diabetes [5]. Risk estimation for individuals aged $<40$ and $>65$ years was performed referring to the risk estimates provided for individuals aged 40 and 65 years, respectively.

\section{Endpoints}

The primary outcome measure was CVD death at 10 years. Additional secondary outcome measures were all-cause death at 10 years and unplanned CVD as well as all-cause hospitalization at 5 years. Time at risk was calculated as time between enrolment and event or end of follow-up period whichever came first. Data on death diagnosis was obtained from the Austrian Death Registry which includes information on cause of death based on ICD-10 codes. CVD events resulting in death or hospitalization were defined as atherosclerotic CVD, valvular heart disease, heart failure, malignant arrhythmia, peripheral artery disease and cerebrovascular disease. In case of unclear ICD-classifications, the hospital charts were further examined to give a definitive diagnosis of cause of death. The adjudication of CVD deaths was carried out by an experienced clinician who was blinded for the various risk assessments.

\section{Statistical analysis}

Continuous data are presented as median and interquartile range (IQR) and discrete data as frequency and percentages. Continuous variables were compared by the Kruskal-Wallis and Mann-Whitney-U-test, counts by the Fisher's exact test.

For the comparison between the risk estimators, i.e. NT-proBNP, ESC/EASD risk model and SCORE, both NT-proBNP and SCORE were entered as continuous as well as categorical variables (NT-proBNP: tertiles and two groups with cut-off: $125 \mathrm{pg} / \mathrm{ml}$; SCORE: 3 risk groups with cut-off: $<5 \%, 5-10 \%,>10 \%)$. All comparisons were made for the total cohort and two subgroups similar to the characteristics of the original derivation cohort of SCORE. For the first subgroup individuals with established CVD were excluded and the second subgroup consisted of patients without established CVD and an age $\geq 40$ and $<65$ years. For outcome analysis we used a cause-specific hazard model. Cox regression was performed to evaluate the association of the risk assessments with 10-year fatal CVD events and secondary outcome measures. Hazard ratios (HRs) of continuous NT-proBNP refer to ln-transformed NTproBNP per 1-IQR increase. In addition to the univariate analysis, adjustments for potential confounders were conducted to demonstrate the robustness of NTproBNP. Albumin/creatinine ratio, eGFR and age and body mass index (BMI) were added as continuous variables to the Cox multivariate regression model, while smoking status, hypertension, dyslipidemia, baseline CVD and sex were added as dichotomous variables (yes/no). Proportional hazard assumption was assessed and satisfied for all variables based on time interaction tests. Cumulative incidence plots of the events of interest are shown for the various risk assessments.

Additionally, we performed a competing risk analysis for the Cox regression model for CVD and non-CVD death as competing risks based on the data duplication method introduced by Lunn and McNeil [28]. Proportional hazard assumption for all variables in the augmented data set was assessed and satisfied based on time interaction tests. Therefore, competing risk analysis was performed by using the event variable as a covariate.

Predictive performance was expressed as discrimination (receiver operating characteristic [ROC] curve, Harrell's C-index) and calibration using HosmerLemeshow goodness-of-fit test. Observed 10-year risk for fatal CVD is presented using the Kaplan-Meier estimates. Differences in outcomes were assessed by nonoverlapping confidence intervals (confidence interval [CI] 95\%) between C-indices corresponding to $\mathrm{p}$-values of $\leq 0.05$.

An improvement in individual risk prediction for the risk assessments was examined by the overall continuous net reclassification improvement (NRI) and presented as NRI (standard error [SE], p-value) as described by Pencina et al. [29] For calculation of the NRI, the ESC/ EASD risk stratification was treated as a categorical variable (moderate, high, very high), the SCORE risk model and NT-proBNP as continuous variables. Furthermore, risk classification tables for all reported outcomes (i.e. allcause mortality, CVD mortality, all-cause hospitalization, CVD hospitalization) were presented comparing SCORE categories and the ESC/EASD risk model with tertiles of NT-proBNP. A two-tailed p-value lower than 0.05 was considered statistically significant. Statistical analysis was performed using SPSS software (IBM SPSS, Chicago, Illinois, USA) version 24, RStudio ( $\mathrm{R}$ Foundation for 
Statistical Computing, Vienna, Austria) version 1.3.1073 and STATA software (StataCorp, College Station, Texas, USA) version 13.

\section{Results}

\section{Study population}

A total of 2186 T2DM patients were enrolled in the study, 496 patients were excluded from the analysis as survival status $(n=460)$ and diabetes duration $(n=36)$ was not available, thus a total of 1690 T2DM patients were analyzed. Detailed description of the baseline characteristics is displayed in Table 1.

Median age of the total study population was 63 years (IQR 54-69), 783 (46\%) of the patients were female. CVD was present in 311 patients(18.4\%). According to the ESC/EASD risk model criteria, 25 (1.5\%) were classified as moderate, 252 (14.9\%) as high and 1125 (66.6\%) as very high risk. A total of 288 patients (17.0\%) were not classifiable based on the stated ESC/EASD criteria, as 280 patients had diabetes duration $<10$ years with 1 or 2 (but $<3$ ) established CV risk factors and 8 patients

Table 1 Baseline characteristics of the overall study cohort

\begin{tabular}{|c|c|}
\hline Characteristics & Overall cohort $(n=1690)$ \\
\hline \multicolumn{2}{|l|}{ Demographics } \\
\hline Age, years (IQR) & $63[54-69]$ \\
\hline Female, n (\%) & $783(46)$ \\
\hline Diabetes duration, years (IQR) & $10[5-19]$ \\
\hline Hypertension, n (\%) & $1135(67)$ \\
\hline Dyslipidaemia, n (\%) & $1152(68)$ \\
\hline Smoking, n (\%) & $339(20)$ \\
\hline $\mathrm{BMI}, \mathrm{kg} / \mathrm{m}^{2}$ (IQR) & $28.7[25.4-32.7]$ \\
\hline \multicolumn{2}{|l|}{ Cardiovascular disease } \\
\hline $\mathrm{PCl}, \mathrm{n}(\%)$ & $66(4)$ \\
\hline $\mathrm{PAD}, \mathrm{n}(\%)$ & $173(10)$ \\
\hline CeVD, n (\%) & $99(6)$ \\
\hline CABG, n (\%) & $62(4)$ \\
\hline \multicolumn{2}{|l|}{ Medications } \\
\hline Statins, n (\%) & $764(45)$ \\
\hline Acetylsalicylic acid, n (\%) & $634(38)$ \\
\hline Insulin, n (\%) & $888(53)$ \\
\hline Oral antidiabetics, n (\%) & $984(58)$ \\
\hline \multicolumn{2}{|l|}{ Laboratory parameters } \\
\hline NT-proBNP, pg/ml (IQR) & 122 [59-266] \\
\hline Albumin/creatinine ratio, mg/mmol (IQR) & $0.87[0.35-2.94]$ \\
\hline $\mathrm{eGFR}, \mathrm{ml} / \mathrm{min}(\mathrm{IQR})$ & $72.7[60.3-85.3]$ \\
\hline LDL cholesterol, mg/dl (IQR) & 102 [82-123] \\
\hline $\mathrm{HbA} 1 \mathrm{c}, \%(\mathrm{IQR})$ & $7.2[6.5-8.1]$ \\
\hline
\end{tabular}

$B M I$ body mass index, $P C l$ percutaneous coronary intervention, $P A D$ peripheral artery disease, CeVD cerebrovascular disease, $C A B G$ coronary artery bypass graft, NT-proBNP N-terminal pro-B-type natriuretic peptide, eGFR estimated glomerular filtration rate, $L D L$ low-density lipoprotein presented with a diabetes duration longer than 10 years without any risk factors. Detailed characteristics for the ESC/EASD risk strata are presented in Additional file 1: Table S3. In the overall cohort, 654 patients (39\%) had a calculated SCORE risk estimate below 5\%, 525 patients (31\%) between 5 and $10 \%$ and 511 patients (30\%) above $10 \%$. The calculated SCORE risk estimates for 10-year fatal increased with ESC/EASD risk category ( $0 \%$ [IQR $0-0$ ] vs. $6 \%$ [IQR 2-10] vs. $8 \%$ [IQR 4-12], p $<0.001$ between all groups).

Distribution of patients in the ESC/EASD and SCORE risk strata as well as proportion of patients with normal $(\mathrm{n}=871)$ and elevated $(\mathrm{n}=819)$ NT-proBNP levels at a cut-off $125 \mathrm{pg} / \mathrm{ml}$ within these groups are illustrated in Fig. 1 ( $\mathrm{p}<0.001$ for both models).

\section{Association of the ESC/EASD risk strata, NT-proBNP and SCORE with the primary endpoint CVD death and the secondary outcome all-cause death at 10 years}

During 10 years of follow-up, 448 patients (26.5\%) died, CVD death accounted to $44.9 \%(n=201)$ of all deaths. The cumulative incidences for all risk models stratified into three groups with regards to both endpoints are shown in Fig. 2. SCORE and NT-proBNP were both significantly associated with the primary outcome CVD death and the secondary outcome all-cause death ( $<<0.001$ for both) while the ESC/EASD risk model was only associated with all-cause death (moderate risk vs. high risk: $\mathrm{p}=0.046$ and vs. very high risk: $\mathrm{p}=0.031$ ). Table 2 shows the results of the univariate Cox regression analysis.

Patients with NT-proBNP $>125 \mathrm{pg} / \mathrm{ml}$ had a 7.2fold and 3.1-fold risk for CVD and all-cause death at 10 years, respectively, compared to individuals with NT-proBNP within the range considered as normal $(\mathrm{p}<0.001)$. NT-proBNP remained a strong predictor of risk irrespective of traditional confounders as age, eGFR, sex, hypertension, smoking, dyslipidaemia, BMI, baseline CVD and albumin/creatinine ratio for both CVD death $(\ln [N T$-proBNP per IQR increase] adjusted HR: 3.96 [2.93-5.35], p <0.001) and all-cause death $(\ln [\mathrm{NT}$-proBNP per IQR increase] adjusted HR: 2.25 [1.85-2.73], $\mathrm{p}<0.001)$.

Competing risk analysis showed qualitatively the same results as in the cause-specific hazard models presented in Table 2. In contrast to SCORE and the ESC/EASD risk model, we found a significant stronger predictive power of NT-proBNP for CVD death than for non-CVD death. Additional file 1: Table S4 provides detailed information on the competing risk analysis. 


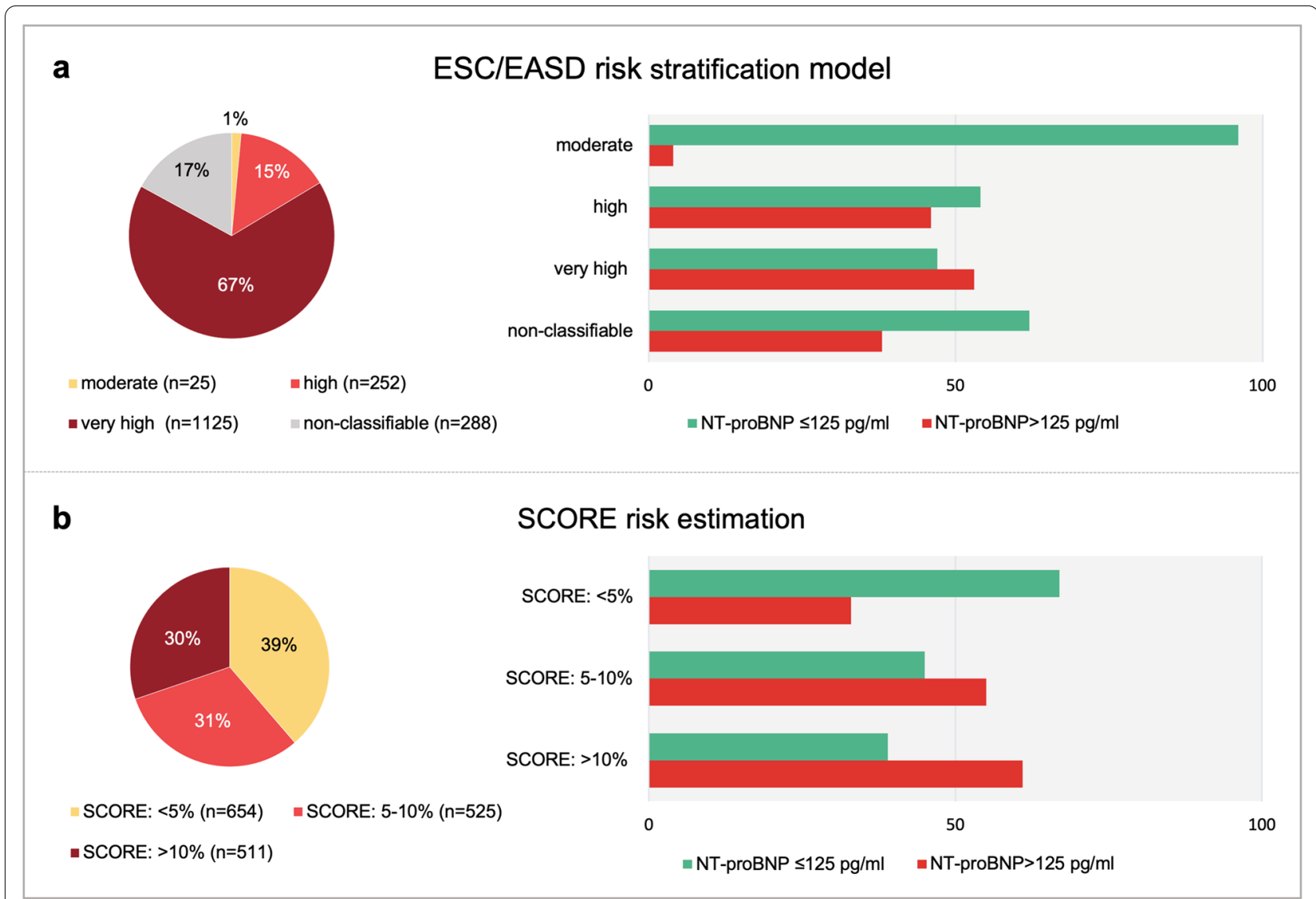

Fig. 1 Distribution of the risk estimate as well as the proportion of patients with normal and elevated NT-proBNP (cut-off: 125 pg/ml) for a the ESC/ EASD risk model and $\mathbf{b}$ SCORE

\section{Discriminatory performance of the ESC/EASD risk strata, NT-proBNP and SCORE in unselected T2DM patients}

ROC curves for all risk models and the endpoints 10-year CVD and all-cause death are shown in Fig. 3. Additional file 1: Table S5 provides information on sensitivity, specifity, negative predictive value and positive predictive value of the respective risk assessments. In terms of discriminatory accuracy NT-proBNP was superior to the ESC/EASD risk model for both outcomes (C-index: CVD death: 0.80 vs. $0.53, \mathrm{p}<0.001$; all-cause death: 0.73 vs. $0.52, \mathrm{p}<0.001$ ). When NT-proBNP was entered as a categorical variable based on tertiles (1st tertile: $59 \mathrm{pg} / \mathrm{mL}$ [IQR 59-59], 2nd tertile: $122 \mathrm{pg} / \mathrm{mL}$ [IQR 90-156], 3rd tertile: $376 \mathrm{pg} / \mathrm{mL}$ [IQR 267-648]) the results remained virtually unchanged (C-index: CVD death: 0.75 vs. 0.53, $\mathrm{p}<0.001$; all-cause death: 0.70 vs. $0.52, \mathrm{p}<0.001)$. Similarly, SCORE showed significantly higher $\mathrm{C}$-indices as compared to the ESC/EASD risk model (C-index: CVD death: 0.64 vs. $0.53, \mathrm{p}=0.001$; all-cause death: 0.66 vs. $0.52, \mathrm{p}<0.001)$. Net individual risk prediction significantly improved when assessing NTproBNP with the ESC/EASD model with a continuous overall NRI of 0.61 (SE 0.60, $\mathrm{p}<0.001$ ) for all-cause death and of 0.74 (SE 0.08, p <0.001) for CVD mortality. When assessing SCORE with the ESC/EASD model, net individual risk prediction did not improve with a continuous overall NRI of 0.12 (SE $0.08, p=0.133$ ) for CVD mortality and 0.06 (SE 0.06, $\mathrm{p}=0.304$ ) for all-cause mortality.

Furthermore, a detailed reclassification table comparing NT-proBNP tertiles with the ESC/EASD model as well as the SCORE model for all presented outcomes is given in Additional file 1: Table S6.

Additional file 1: Table S7 provides information on the predictive performance of NT-proBNP when added to a baseline model encompassing classical CV risk factors. Also here, NT-proBNP provided prognostic information beyond traditional risk factors. 


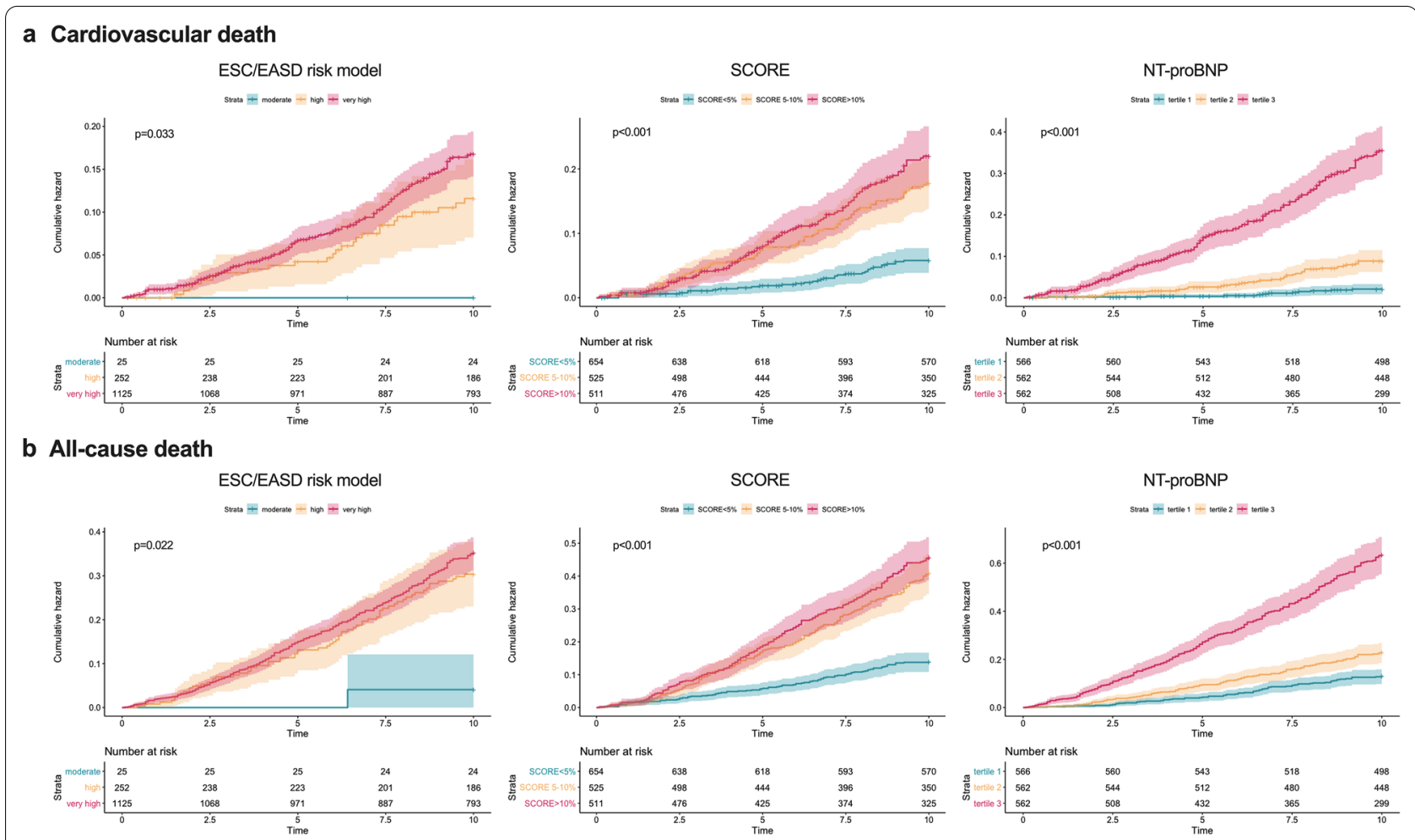

Fig. 2 Kaplan-Meier curves showing the cumulative incidence of (a) cardiovascular and (b) all-cause death for the ESC/EASD risk model (left), SCORE (cut-off: $<5 \%, 5-10 \%,>10 \%$ ) (middle), and NT-proBNP tertiles (right)

Table 2 Association of the ESC/EASD model, NT-proBNP and SCORE with outcome in unselected patients with T2DM $(n=1690)$

\begin{tabular}{|c|c|c|c|c|c|c|}
\hline & \multicolumn{3}{|c|}{ 10-years cardiovascular death } & \multicolumn{3}{|c|}{ 10-years all-cause death } \\
\hline & $\mathrm{HR}[95 \% \mathrm{Cl}]$ & $\mathbf{P}$ & C-index $[95 \% \mathrm{Cl}]$ & $\mathrm{HR}[95 \% \mathrm{Cl}]$ & $\mathbf{P}$ & C-index $[95 \% \mathrm{Cl}]$ \\
\hline ESC/EASD risk model & - & - & $0.53[0.50$ to 0.56$]$ & - & & $0.52[0.50$ to 0.54$]$ \\
\hline Moderate & Reference & - & - & Reference & - & - \\
\hline High & $23.68[0.08-7013.72]$ & 0.276 & - & $7.49[1.04-53.94]$ & 0.046 & - \\
\hline Very high & $20.88[0.25-1747.39]$ & 0.178 & - & $8.63[1.21-61.42]$ & 0.031 & - \\
\hline SCORE, $\%^{\mathrm{a}}(<5 \%, 5-10 \%,>10 \%)$ & - & - & $0.63[0.60-0.67]$ & - & - & $0.62[0.60-0.64]$ \\
\hline$<5 \%$ & Reference & - & - & Reference & - & - \\
\hline $5-10 \%$ & $3.10[2.08-4.63]$ & $<0.001$ & - & $2.90[2.24-3.76]$ & $<0.001$ & - \\
\hline$<10 \%$ & $3.79[2.56-5.60]$ & $<0.001$ & - & $3.25[2.52-4.21]$ & $<0.001$ & - \\
\hline SCORE, $\%$ & $1.05[1.04-1.07]$ & $<0.001$ & $0.64[0.60-0.67]$ & $1.06[1.04-1.07]$ & $<0.001$ & $0.66[0.63-0.68]$ \\
\hline NT-proBNP, tertiles & - & - & $0.75[0.73-0.78]$ & - & - & $0.70[0.67-0.72]$ \\
\hline Tertile 1 & Reference & - & - & Reference & - & - \\
\hline Tertile 2 & $4.24[2.19-8.21]$ & $<0.001$ & - & $1.76[1.31-2.38]$ & $<0.001$ & - \\
\hline Tertile 3 & $17.21[9.3-31.78]$ & $<0.001$ & - & $4.90[3.76-6.39]$ & $<0.001$ & - \\
\hline NT-proBNP, pg/ml ${ }^{b}$ & $5.72[4.68-7.00]$ & $<0.001$ & $0.80[0.77-0.83]$ & 3.32 [2.90-3.79] & $<0.001$ & $0.73[0.70-0.76]$ \\
\hline NT-proBNP, > $125 \mathrm{pg} / \mathrm{ml}$ & 7.15 [4.85-10.53] & $<0.001$ & $0.71[0.68-0.74]$ & $3.14[2.56-3.86]$ & $<0.001$ & $0.66[0.63-0.68]$ \\
\hline
\end{tabular}

a Refers to SCORE treated as categorical variable (cut-off: $<5 \%, 5-10 \%,>10 \%$ )

${ }^{b}$ Refers to In-transformed NT-proBNP per 1-IQR increase 


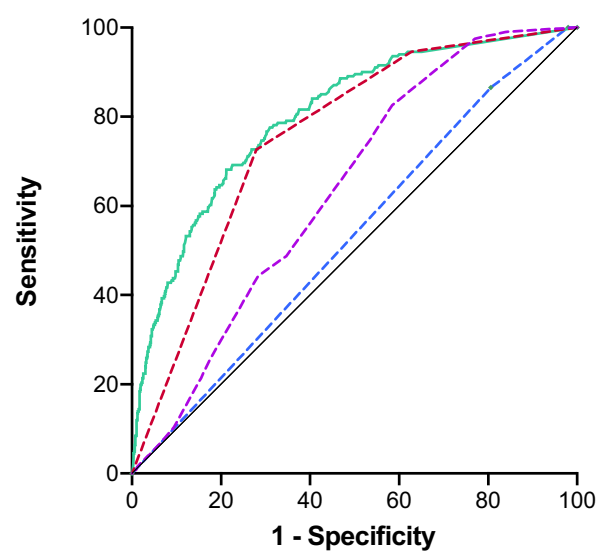

b All-cause death

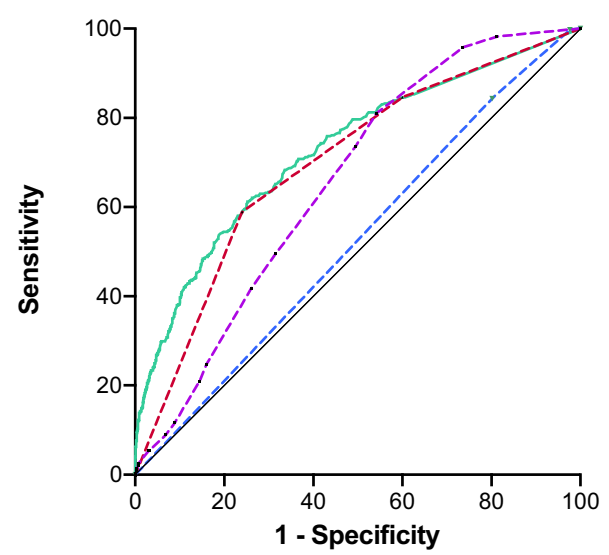

NT-proBNP - - NT-proBNP tertile - - SCORE - - ESC/EASD risk stratification

Fig. 3 Receiver operating characteristic curves of the ESC/EASD risk model, NT-proBNP and SCORE for the outcomes (a) cardiovascular death and (b) all-cause death

Predictive performance of the ESC/EASD risk strata, NT-proBNP and SCORE in distinct subgroups

The following subgroups were investigated: (i) patients without CVD and (ii) patients without CVD and aged 40-64 years according to SCORE derivation cohort.

Cox regression analysis for the subgroups regarding 10-year fatal outcome are shown in Additional file 1: Table S8. NT-proBNP and SCORE were equally associated with 10-year CVD and all-cause death in subgroups of patients without established CVD and in patients without CVD and aged between 40 and 64 years $(\mathrm{p}<0.001$ for all). The ESC/EASD risk model was only associated with 10-year all-cause death in patients without CVD when comparing the moderate risk with the high risk category $(\mathrm{p}=0.046)$.

Figure 4 displays C-statistics of the risk assessments according to the T2DM population studied, the respective ROC graphics are shown in Additional file 1: Figure S1. NT-proBNP was characterized by robustly highest $\mathrm{C}$-indices across both subgroups comparable to the unselected cohort for both endpoints. The ESC/ EASD model was characterized by poor $\mathrm{C}$-indices in all groups. The performance of the SCORE risk prediction model improved with progressing exclusivity of patient criteria, performing best in the cohort closest to its derivation population, i.e. in patients without CVD and aged 40-64 years. Both NT-proBNP and SCORE outperformed the ESC/EASD model with regards to CVD death and all-cause death in the subgroup without CVD while only NT-proBNP achieved a significantly higher C-index to predict CVD death in the subgroup including patients without CVD and aged 40-64 years.

\section{Observed versus predicted risk estimate for 10-year fatal CVD by SCORE}

The SCORE risk algorithm underestimated the actual risk of CVD death in unselected T2DM patients (observed vs. predicted CVD fatal risk: $13 \%$ vs. $8 \%$ [IQR 4-12]; $\left.\mathrm{X}^{2}=70.1, \mathrm{p}<0.001\right)$ and in T2DM patients without CVD (observed vs. predicted CVD fatal risk: $10 \%$ vs. $8 \%$ [IQR $\left.4-12] ; X^{2}=19.8, p<0.001\right)$. When investigating only individuals without CVD and aged 40-64 years, the observed risk was $6 \%$ compared with a median predicted risk of $4 \%$ [IQR 2-8]. Here, goodness-of-fit for SCORE risk estimate was good with a $\mathrm{X}^{2}$ of $4.3(\mathrm{p}=0.234)$.

\section{Predictive performance of the ESC/EASD risk strata, NT-proBNP and SCORE for the secondary outcomes 5-year CVD and all-cause hospitalization}

Over a follow-up of 5 years, 1053 (62.3\%) patients were hospitalized due to any causes and 367 (21.7\%) patients due to unplanned CVD events. Risk for all-cause hospitalization increased by $7 \%$ and for CVD hospitalization by $12 \%$ per $100 \mathrm{pg} / \mathrm{ml}$ increase in NT-proBNP $(\mathrm{p}<0.001$ for both). Similarly, SCORE was associated with increased risk for CVD and all-cause hospitalizations $(\mathrm{p}<0.001$ for both) while the ESC/EASD risk stratification model was only associated with all-cause 






hospitalizations (moderate risk vs. high risk: $\mathrm{p}=0.033$ and vs. very high risk: $\mathrm{p}=0.002$ ). Cox regression analysis is presented in Additional file 1: Table S9. Figure 5 presents cumulative incidence of 5-year hospitalizations for NT-proBNP. In terms of discriminatory accuracy NT-proBNP was superior to the ESC/EASD risk model for both outcomes (C-index: CVD hospitalization: 0.74 vs. 0.54 ; all-cause hospitalization: 0.62 vs. $0.55 ; \mathrm{p}<0.001$ for all comparisons). In comparison to the ESC/EASD risk model, SCORE showed significantly higher C-indices for CVD-hospitalizations (0.62 vs.
$0.54, \mathrm{p}=0.003)$ and for all-cause hospitalizations $(0.59$ vs. $0.55, \mathrm{p}=0.040$ ).

The NRI significantly improved when assessing NT-proBNP with the ESC/EASD model with a continuous overall NRI of 0.32 (SE 0.06, $\mathrm{p}<0.001$ ) for allcause hospitalizations and of 0.70 (SE 0.06, p < 0.001) for CVD hospitalizations. Similarly, when assessing SCORE with the ESC/EASD model, net individual risk prediction significantly improved with a continuous overall NRI of 0.15 (SE $0.06, p=0.008$ ) for all-cause 
hospitalizations and of $0.14(\mathrm{SE} 0.06, \mathrm{p}=0.024)$ for CVD hospitalizations.

\section{Outcome specifity of NT-proBNP, the ESC/EASD risk strata and SCORE}

As indicated by C-statistics, NT-proBNP yielded better discrimination for CVD than for all-cause death (0.80 vs. 0.73, $\mathrm{p} \leq 0.05)$ and for CVD than for all-cause hospitalization (0.74 vs. $0.62, \mathrm{p} \leq 0.05)$. No difference for the $\mathrm{ESC} /$ EASD risk strata (CVD vs. all-cause death: 0.53 vs. 0.52 , $\mathrm{p}>0.05$; CVD vs all-cause hospitalization: 0.54 vs. 0.55 , $\mathrm{p}>0.05$ ) or the SCORE risk estimation (CVD vs. all-cause death: 0.64 vs. $0.66, \mathrm{p}>0.05$; CVD vs all-cause hospitalization: 0.62 vs. $0.59, \mathrm{p}>0.05$ ) could be observed.

\section{Discussion}

This is the first study evaluating the predictive performance of the recently published ESC/EASD risk stratification model in a reasonably large real-world cohort of patients with T2DM and directly comparing this risk model with SCORE and the biomarker NT-proBNP. Our results demonstrate that (i) the ESC/EASD risk stratification model performs limited compared to SCORE and NT-proBNP in terms of risk prediction and discriminatory accuracy (ii) application of SCORE in a selected subgroup of T2DM patients resulted in a similar discriminative ability as achieved in non-diabetics, (iii) in both unselected and selected T2DM patients NT-proBNP remains a robust predictor for outcome, (iv) in contrast to NT-proBNP the ESC/EASD and SCORE risk model showed no outcome specifity for future CVD events in T2DM individuals.

The 2016 ESC guidelines on CVD prevention classify most patients with T2DM at high or very high risk [4]. Although diabetes has long been considered as a "cardiovascular risk equivalent" [30], more recent data indicate that incremental CV risk does not uniformly affect all patients with T2DM [31, 32]. Therefore, tools advocating a more individualized risk assessment are mandatory. The recently updated guidelines for the management and prevention of CVD risk in individuals with diabetes integrated the aforementioned approach and introduced a new risk model accounting also for individuals at moderate risk [3]. The new guidelines recommend for the first time the use of a risk stratification model based on three risk categories (moderate/high/very high) to aid treatment decisions in diabetes while the assessment of biomarkers (e.g. NT-proBNP) is not recommended. However, the predictive performance of the ESC/EASD has neither been derived nor tested in patients with diabetes.

When applying the ESC/EASD risk criteria to our cohort, most patients with $67 \%$ were stratified to the very high risk category, $15 \%$ met the ESC/EASD criteria for the high risk category whereas the moderate risk category was poorly represented with $1.5 \%$. Moreover, a significant proportion of patients $(17 \%)$ could not be categorized into either of the ESC/EASD CVD risk categories based on the model's stratification criteria. Apparently, the newly defined moderate risk category, determined by short diabetes duration and no risk factors at all, is poorly represented in a typical cohort of patients with T2DM. The high prevalence of risk factors such as obesity, hypertension and dyslipidemia even in individuals with short T2DM duration (as part of the metabolic syndrome) may explain the small number of patients stratified into this group [33]. Moreover, our data indicate that the ESC/ EASD risk model does not provide an accurate risk estimate to predict 10-year fatal CVD outcome in individuals with diabetes. In direct comparison, both NT-proBNP and SCORE outperformed the ESC/EASD model in terms of risk prediction and discriminatory accuracy.

SCORE based risk estimation has been developed in the general population, but notably the original derivation cohort included also diabetic patients [5]. Since data on diabetes has not been collected uniformly in the SCORE project, the presence of diabetes has not been included as a predictor variable in the risk algorithm. However, it has been suggested that SCORE could be used for a rough risk assessment in patients with diabetes. In the current report, SCORE was significantly associated with 10-year risk of fatal CVD but underestimated the risk for fatal CVD events in patients with T2DM. Of note, agreement between observed and predicted risk improved in the subgroup closest to the derivation cohort of SCORE, i.e. patients without CVD and aged between 40 and 64 years. Similarly, the discriminatory ability for 10 -years CVD death improved in this subgroup with a C-index of 0.69, which is similar to SCORE in non-diabetics [5]; however, resulted in the exclusion of more than half of the study population. Since T2DM is particularly a disease of the elderly, age restrictions as given by SCORE would limit its utility in clinical practice.

Previous studies reported that CVD risk scores developed in the general population underestimate risk in individuals with T2DM [34]. Conversely, a brief report by Coleman et al. investigating SCORE in 3898 individuals with newly diagnosed T2DM from the UKPDS cohort reported that SCORE risk equation overestimates the 10 -year risk for fatal CVD in individuals with T2DM by $18 \%$, but provides good discriminatory accuracy with a C-index of 0.77 for fatal CVD events [6]. However, the direct comparison of these results with our data may be limited, as the UKPDS included only individuals with newly diagnosed T2DM and treatment has been significantly changed within the study period. 
Although much of CV risk can be attributed to traditional risk factors incorporated in classical risk prediction models [35], they do not explain the full spectrum of CVD risk in diabetes [36, 37]. Potential limitations of risk scores calculated at a single point in time may be their inability to account for variability in measured risk factors (e.g. blood pressure), biological variation, exposure duration or untreated risk factor severity. It is not surprising, then, that major $\mathrm{CV}$ risk factors are also highly prevalent among individuals who will never experience a CVD event [37]. This paradoxon points to the need for risk assessment tools that allow a more integrated approach at the individual patient level. Intimately tied to this effort will be the requirement to individualize risk beyond the presence of established risk factors.

The current report demonstrates that single NTproBNP measurement provides a more accurate risk estimate than the newly introduced ESC/EASD risk model. In contrast to both the ESC/EASD model and SCORE, NT-proBNP was even more specific for increased CV risk than all-cause risk in T2DM, emphasizing its clinical relevance as an outcome specific marker.

Yet, numerous studies have indicated that NT-proBNP assessment is effective in refining risk prediction for CVD and adds predictive power to conventional risk models in individuals with T2DM $[7-17,20]$, only a few studies investigated single NT-proBNP in direct comparison to models composing multiple risk variables [16, 17]. In daily clinical practice, the assessment of a single marker that allows to identify those at highest risk seems attractive. In this study, as in others [18-22], NT-proBNP levels above $125 \mathrm{pg} / \mathrm{ml}$ were strongly associated with adverse outcomes in T2DM patients. Given the high negative predictive value of NT-proBNP at a cutoff level of $125 \mathrm{mg} / \mathrm{dl}$ [21, 22], initial assessment of NT-proBNP could serve as a first-line screening tool that allows to safely and effectively rule out increased CV risk, while higher values would require further evaluation. In a second approach, additional cardiac investigations may be applied to further refine individual risk. A recent published study conducted in asymptomatic individuals with T2DM reported an additive predictive value of NTproBNP combined with coronary artery calcium scoring [24]. Similarly, several other studies reported incremental prognostic information of NT-proBNP and troponin $\mathrm{T}$ when used in combination [13-15, 23].

The association of NT-proBNP with (CV) hospital admissions observed in this report indicates that NTproBNP-guided risk stratification may also have the potential for overall cost reductions as already exemplified by previous natriuretic-guided trials in heart failure [38]. The use of NT-proBNP would omit the need for calculation of scores as well as the problem of nonclassification, misclassification or overfitting observed in global risk estimation models.

Yet, two trials provided initial evidence on the effectiveness of natriuretic peptides in guiding preventive efforts in patients at high risk for developing CVD events $[19,39]$. In the prospective randomized controlled PONTIAC trial (NT-proBNP selected prevention of cardiac events in a population of diabetic patients without a history of cardiac disease) measurement of NT-proBNP (cut-off $125 \mathrm{pg} / \mathrm{ml}$ ) was used to identify T2DM patients at high risk for developing CVD [19]. These patients were then stratified to either standard of care treatment or titration for renin-angiotensin inhibitors and betablockers. A significant reduction of CVD events was reported in the treatment arm providing initial evidence for a NT-proBNP measurement-based selection of highrisk individuals with T2DM. Similarly, the STOP-HF study (St. Vincent's Screening to Prevent Heart Failure) demonstrated the effectiveness of natriuretic peptides in guiding preventive efforts in patients with various $\mathrm{CV}$ risk factors [39]. A recent observation from the CANVAS (Canagliflozin Cardiovascular Assessment Study) study has shown that canagliflozin treatment in T2DM patients with NT-proBNP levels above $125 \mathrm{pg} / \mathrm{ml}$ achieved greater absolute risk reductions in event rates compared to those with lower concentrations [18].

In general, screening for high-risk individuals may only be appropriate when effective treatments are available. The high prevalence of modifiable risk factors among individuals with diabetes but also the recent emerge of new therapies with favorable effects on CVD outcome, such as sodium-glucose cotransporter-2 inhibitors, underscore the importance of identifying those who would most probably benefit from initiation or optimizing treatment.

\section{Limitations}

We are aware of the following limitations of our study. First, retinopathy was not generally documented in this registry and could therefore not be implemented in the risk score which could have led to misclassification. In the current report only $16 \%$ of the patients were categorized as moderate or high risk. Hypothetically, the eventual identification of individuals with retinopathy would have led to even more patients being stratified into the very high-risk group, resulting in an even greater weighting of the very high-risk category. A study by Klein et al. demonstrated that retinopathy occurs more frequently in patients with long-term diabetes, CVD and proteinuria [40]. Given the very high risk criteria of the ESC/EASD risk model it seems conceivable that patients with retinopathy may also have been captured in the very highrisk category. Second, as this registry included mainly 
outpatients followed in hospital, T2DM individuals at lower risk who are more often treated by general practitioners might be underrepresented. In this context, the ESC/EASD model might have performed different with an altered cohort including these patients. Third, survival status was not available in 460 patients after 10 years follow-up. Last, an inverse association between circulating levels of natriuretic peptides and BMI has been reported earlier [41], which could be a potential limitation in a population of T2DM patients. However, despite these observations, natriuretic peptides have been shown to retain prognostic performance in obese patients [42].

\section{Conclusion}

Overall, the current report shows that the recently introduced ESC/EASD risk stratification model provides only limited prognostic information in direct comparison to SCORE and most notably to single NT-proBNP assessment. NT-proBNP measurement is a simple and independent screening tool to identify individuals at increased risk for specifically adverse CVD outcome applicable in a broad spectrum of T2DM patients. Future studies need to investigate the cost-effectiveness and feasibility of NT-proBNP-based screening.

\section{Supplementary Information}

The online version contains supplementary material available at https://doi. org/10.1186/s12933-021-01221-w.



\section{Abbreviations}

BMI: Body mass index; Cl: Confidence interval; CV: Cardiovascular risk; CVD: Cardiovascular disease; EASD: European Association for the Society of Diabetes; eGFR: Estimated glomerular filtration rate; ESC: European Society of Cardiology; HR: Hazard ratio; IQR: Interquartile range; NRI: Net reclassification improvement; NT-proBNP: N-terminal pro-B-type natriuretic peptide; ROC:
Receiver operating characteristic; SCORE: Systemic COronary Risk Evaluation; SE: Standard error; T2DM: Type 2 diabetes mellitus.

\section{Acknowledgements}

Not applicable.

\section{Authors' contributions}

HA, MC, MR, SP, NP, GG, RW, MH and PEB contributed to the conception or design of the work. GS, MC, MR, SN, SP, GG, NP and MH contributed to the acquisition, analysis, or interpretation of data for the work. HA, MC, MR, SN, SP, GG, NP, RW, MH and PEB drafted the manuscript. MR, SP, NP, GG, RW and $\mathrm{MH}$ critically revised the manuscript. All gave final approval and agree to be accountable for all aspects of work ensuring integrity and accuracy. All authors read and approved the final manuscript

\section{Funding}

This work was supported by the grant from the Austrian Science Fund [KLI 700-B30].

\section{Availability of data and materials}

The authors declare that all data supporting the findings of this study are available within the article and its supplementary information files.

\section{Ethics approval and consent to participate}

All patients gave written informed consent and the study protocol was approved by the Ethics Committee of the Medical University of Vienna.

\section{Consent for publication}

Not applicable.

\section{Competing interests \\ None declared.}

\section{Author details}

1 Department of Internal Medicine II, Medical University of Vienna, Waehringer Guertel 18-20, 1090 Vienna, Austria. ${ }^{2}$ Department of Internal Medicine, Saint John of God Hospital Linz, Seilerstaette 2, 4021 Linz, Austria. ${ }^{3}$ Department of Medicine IV, Clinic Favoriten, Kundratstraße 3, 1100 Vienna, Austria. ${ }^{4}$ Complexity Research, Schönbrunner Straße 32, 1050 Vienna, Austria.

Received: 10 August 2020 Accepted: 20 January 2021

Published online: 02 February 2021

\section{References}

1. Einarson TR, Acs A, Ludwig C, Panton UH. Prevalence of cardiovascular disease in type 2 diabetes: a systematic literature review of scientific evidence from across the world in 2007-2017. Cardiovasc Diabetol. 2018;17(1):83.

2. Bertoluci MC, Rocha VZ. Cardiovascular risk assessment in patients with diabetes. Diabetol Metab Syndr. 2017;9(1):1-13.

3. Cosentino F, Grant PJ, Aboyans V, Bailey CJ, Ceriello A, Delgado V, et al. 2019 ESC Guidelines on diabetes, pre-diabetes, and cardiovascular diseases developed in collaboration with the EASD. Eur Heart J. 2020;41(2):255-323.

4. Piepoli MF, Hoes AW, Agewall S, Albus C, Brotons C, Catapano AL, et al. 2016 European Guidelines on cardiovascular disease prevention in clinical practice. Eur Heart J. 2016;37(29):2315-81.

5. Conroy RM, Pyörälä K, Fitzgerald AP, Sans S, Menotti A, De Backer G, et al. Estimation of ten-year risk of fatal cardiovascular disease in Europe: the SCORE project. Eur Heart J. 2003;24(11):987-1003.

6. Coleman RL, Stevens RJ, Retnakaran R, Holman RR. Framingham, SCORE, and DECODE risk equations do not provide reliable cardiovascular risk estimates in type 2 diabetes. Diabetes Care. 2007;30(5):1292-4.

7. Ballo P, Betti I, Barchielli A, Balzi D, Castelli G, De Luca L, et al. Prognostic role of $\mathrm{N}$-terminal pro-brain natriuretic peptide in asymptomatic hypertensive and diabetic patients in primary care: impact of age and gender: results from the PROBE-HF study. Clin Res Cardiol. 2016;105(5):421-31.

8. Price AH, Welsh P, Weir CJ, Feinkohl I, Robertson CM, Morling JR, et al. $\mathrm{N}$-terminal pro-brain natriuretic peptide and risk of cardiovascular events 
in older patients with type 2 diabetes: the Edinburgh type 2 diabetes study. Diabetologia. 2014;57(12):2505-12.

9. Huth C, Bauer A, Zierer A, Sudduth-Klinger J, Meisinger C, Roden M, et al. Biomarker-defined pathways for incident type 2 diabetes and coronary heart disease - a comparison in the MONICA/KORA study. Cardiovasc Diabetol. 2020;19(1):32.

10. Scirica BM, Bhatt DL, Braunwald E, Raz I, Cavender MA, Im K, et al. Prognostic implications of biomarker assessments in patients with type 2 diabetes at high cardiovascular risk: a secondary analysis of a randomized clinical trial. JAMA Cardiol. 2016;1 (9):989-98.

11. Looker HC, Colombo M, Agakov F, Zeller T, Groop L, Thorand B, et al. Protein biomarkers for the prediction of cardiovascular disease in type 2 diabetes. Diabetologia. 2015;58(6):1363-71.

12. Sharma A, Vaduganathan M, Ferreira JP, Liu Y, Bakris GL, Cannon CP, et al. Clinical and biomarker predictors of expanded heart failure outcomes in patients with type 2 diabetes mellitus after a recent acute coronary syndrome: insights from the EXAMINE trial. J Am Heart Assoc. 2020;9(1):e012797.

13. Hillis GS, Welsh P, Chalmers J, Perkovic V, Chow CK, Li Q, et al. The relative and combined ability of high-sensitivity cardiac troponin $T$ and $\mathrm{N}$-terminal pro-B-type natriuretic peptide to predict cardiovascular events and death in patients with type 2 diabetes. Diabetes Care. 2014;37(1):295-303.

14. Wong $Y-K$, Cheung CYY, Tang CS, Hai JSH, Lee C-H, Lau K-K, et al. Highsensitivity troponin I and B-type natriuretic peptide biomarkers for prediction of cardiovascular events in patients with coronary artery disease with and without diabetes mellitus. Cardiovasc Diabetol. 2019;18(1):171.

15. Nguyen K, Fan W, Bertoni A, Budoff MJ, Defilippi C, Lombardo D, et al. $\mathrm{N}$-terminal Pro B-type natriuretic peptide and high-sensitivity cardiac troponin as markers for heart failure and cardiovascular disease risks according to glucose status (from the Multi-Ethnic Study of Atherosclerosis [MESA]). Am J Cardiol. 2020;125(8):1194-201.

16. Malachias MVB, Jhund PS, Claggett BL, Wijkman MO, Bentley-Lewis R, Chaturvedi N, et al. NT-proBNP by itself predicts death and cardiovascular events in high-risk patients with type 2 diabetes mellitus. J Am Heart Assoc. 2020;9(19):e017462.

17. Wolsk E, Claggett B, Pfeffer MA, Diaz R, Dickstein K, Gerstein HC, et al. Role of B-type natriuretic peptide and N-terminal prohormone BNP as predictors of cardiovascular morbidity and mortality in patients with a recent coronary event and type 2 diabetes mellitus. J Am Heart Assoc. 2017;6(6):e004743.

18. Januzzi JபJ, Xu J, Li J, Shaw W, Oh R, Pfeifer M, et al. Effects of canagliflozin on amino-terminal Pro-B-type natriuretic peptide: implications for cardiovascular risk reduction. J Am Coll Cardiol. 2020;76(18):2076-85.

19. Huelsmann M, Neuhold S, Resl M, Strunk G, Brath H, Francesconi C, et al. PONTIAC (NT-proBNP selected prevention of cardiac events in a population of diabetic patients without a history of cardiac disease): a prospective randomized controlled trial. J Am Coll Cardiol. 2013;62 (15):1365-72.

20. Gori M, Gupta DK, Claggett B, Selvin E, Folsom AR, Matsushita K, et al. Natriuretic peptide and high-sensitivity troponin for cardiovascular risk prediction in diabetes: the Atherosclerosis Risk in Communities (ARIC) study. Diabetes Care. 2016;39(5):677-85.

21. Huelsmann M, Neuhold S, Strunk G, Moertl D, Berger R, Prager R, et al. NT-proBNP has a high negative predictive value to rule-out short-term cardiovascular events in patients with diabetes mellitus. Eur Heart J. 2008;29(18):2259-64.

22. Betti I, Castelli G, Barchielli A, Beligni C, Boscherini V, De Luca L, et al. The role of $\mathrm{N}$-terminal PRO-brain natriuretic peptide and echocardiography for screening asymptomatic left ventricular dysfunction in a population at high risk for heart failure. The PROBE-HF Study. J Cardiac Fail. 2009;15(5):377-84.

23. Rørth R, Jhund PS, Kristensen SL, Desai AS, Køber L, Rouleau JL, et al. The prognostic value of troponin T and N-terminal pro B-type natriuretic peptide, alone and in combination, in heart failure patients with and without diabetes. Eur J Heart Fail. 2019;21 (1):40-9.

24. von Scholten BJ, Reinhard H, Hansen TW, Lindhardt M, Petersen CL, Wiinberg $\mathrm{N}$, et al. Additive prognostic value of plasma $\mathrm{N}$-terminal pro-brain natriuretic peptide and coronary artery calcification for cardiovascular events and mortality in asymptomatic patients with type 2 diabetes. Cardiovasc Diabetol. 2015;14:59.
25. Bertsch T, Chapelle J-P, Dempfle C-E, Giannitsis E, Schwabs M, Zerback R. Multicentre analytical evaluation of a new point-of-care system for the determination of cardiac and thromboembolic markers. Clin Lab. 2010;56(1-2):37-49.

26. Mach F, Baigent C, Catapano AL, Koskinas KC, Casula M, Badimon L, et al. 2019 ESC/EAS guidelines for the management of dyslipidaemias: lipid modification to reduce cardiovascular risk. Eur Heart J. 2020;41(1):111-88.

27. Williams B, Mancia G, Spiering W, Agabiti Rosei E, Azizi M, Burnier M, et al. 2018 ESC/ESH guidelines for the management of arterial hypertension. Eur Heart J. 2018;39(33):3021-104.

28. Lunn M, McNeil D. Applying Cox regression to competing risks. Biometrics. 1995;51 (2):524-32.

29. Pencina MJ, D'Agostino RBS, D'Agostino RBJ, Vasan RS. Evaluating the added predictive ability of a new marker: from area under the ROC curve to reclassification and beyond. Stat Med. 2008;27(2):112-57.

30. Haffner SM, Lehto S, Rönnemaa T, Pyörälä K, Laakso M. Mortality from coronary heart disease in subjects with type 2 diabetes and in nondiabetic subjects with and without prior myocardial infarction. N Engl J Med. 1998;339(4):229-34

31. Bulugahapitiya U, Siyambalapitiya S, Sithole J, Idris I. Is diabetes a coronary risk equivalent? Systematic review and meta-analysis. Diabet Med. 2009;26(2):142-8.

32. Rana JS, Liu JY, Moffet HH, Jaffe M, Karter AJ. Diabetes and prior coronary heart disease are not necessarily risk equivalent for future coronary heart disease events. J Gen Intern Med. 2016;31(4):387-93.

33. Hillier TA, Pedula KL. Characteristics of an adult population with newly diagnosed type 2 diabetes: the relation of obesity and age of onset. Diabetes Care. 2001;24(9):1522-7.

34. Chamnan P, Simmons RK, Sharp SJ, Griffin SJ, Wareham NJ. Cardiovascular risk assessment scores for people with diabetes: a systematic review. Diabetologia. 2009:52(10):2001-14.

35. Magnus P, Beaglehole R. The real contribution of the major risk factors to the coronary epidemics: time to end the "only-50\%" myth. Arch Intern Med. 2001;161(22):2657-60.

36. Sarwar N, Gao P, Seshasai SRK, Gobin R, Kaptoge S, Di Angelantonio E, et al. Diabetes mellitus, fasting blood glucose concentration, and risk of vascular disease: a collaborative meta-analysis of 102 prospective studies. Lancet. 2010;375(9733):2215-22.

37. Greenland P, Knoll MD, Stamler J, Neaton JD, Dyer AR, Garside DB, et al. Major risk factors as antecedents of fatal and nonfatal coronary heart disease events. JAMA. 2003;290(7):891-7.

38. Sanders-van Wijk S, Van Asselt ADI, Rickli H, Estlinbaum W, Erne P, Rickenbacher $\mathrm{P}$, et al. Cost-effectiveness of $\mathrm{N}$-terminal pro-b-type natriureticguided therapy in elderly heart failure patients. Results from TIME-CHF (Trial of intensified versus standard medical therapy in elderly patients with congestive heart failure). JACC Heart Fail. 2013;1(1):64-71.

39. Ledwidge M, Gallagher J, Conlon C, Tallon E, O'Connell E, Dawkins I, et al. Natriuretic peptide-based screening and collaborative care for heart failure: the STOP-HF randomized trial. JAMA. 2013;310(1):66-74.

40. Klein R, Marino EK, Kuller LH, Polak JF, Tracy RP, Gottdiener JS, et al. The relation of atherosclerotic cardiovascular disease to retinopathy in people with diabetes in the Cardiovascular Health Study. Br J Ophthalmol. 2002:86(1):84-90.

41. Nadruz WJ, Claggett BL, McMurray JJ, Packer M, Zile MR, Rouleau JL, et al. Impact of body mass index on the accuracy of $\mathrm{N}$-terminal Pro-Brain natriuretic peptide and brain natriuretic peptide for predicting outcomes in patients with chronic heart failure and reduced ejection fraction: insights from the PARADIGM-HF Study (prospective comparison of ARNI with ACEI to determine impact on global mortality and morbidity in heart failure trial). Circulation. 2016;134(22):1785-7.

42. Bayes-Genis A, Lloyd-Jones DM, van Kimmenade RRJ, Lainchbury JG, Richards AM, Ordoñez-Llanos J, et al. Effect of body mass index on diagnostic and prognostic usefulness of amino-terminal pro-brain natriuretic peptide in patients with acute dyspnea. Arch Intern Med. 2007;167(4):400-7.

\section{Publisher's Note}

Springer Nature remains neutral with regard to jurisdictional claims in published maps and institutional affiliations. 